\title{
Современное состояние проблемы реконструктивно- восстановительных операций при неопухолевых заболеваниях толстой кишки. Часть 3.
}

\author{
В. М. Мельник, А. И. Пойда, Абдулрахман Абдул Кадир
}

Национальный медицинский университет имени А. А. Богомольца, г. Киев

\section{Modern state of a problem of reconstructive-restoration operations in nontumoral diseases of the large bowel. Part 3.}

\author{
V. M. Melnik, A. I. Poyda, Abdulrakhman Abdul Kadir \\ Bogomolets National Medical University, Kyiv
}

\begin{abstract}
Профилактика, лечение последствий и осложнений реконструктивно-восстановительных операций при неопухолевых заболеваниях толстой кишки.

Тонкокишечные резервуарные конструкции в настоящее время являются единственным эффективным хирургическим пособием для предупреждения тяжелых постколэктомических нарушений, поэтому некоторые авторы считают их обязательной слагающей реконструктивно-восстановительных операций после колэктомии и резекции прямой кишки [1 - 5]. Однако у 24-32\% больных формирование указанных конструкций сопряжено с дополнительными техническими трудностями, обусловленными короткой брыжейкой и особенностями ангиоархитектоники тонкой кишки, что затрудняет дислокацию ее терминального отдела вместе с тонкокишечным резервуаром в полость малого таза, формирование резервуарно-анального анастомоза [4, 6]. В связи с этим большое практическое значение имеет разработка эффективных способов дислокации тонкокишечного трансплантата в полость малого таза.

Кроме формирования тонкокишечного резервуара во время выполнения реконструктивно-восстановительного этапа оперативного вмешательства, чрезвычайно важным фактором хирургической реабилитации больных, оперированных в два этапа по поводу язвенного колита или семейного полипоза толстой кишки, является профилактика постколэктомического синдрома в период между радикальной и реконструктивно-восстановительной операциями $[7,8]$. Указанные двухэтапные операции выполняют у 11 - 78,2\% больных в связи с тяжелым, осложненным течением заболеваний, сопровождающихся выраженными метаболическими нарушениями, высоким риском возникновения осложнений со стороны илеоанального, резервуарно-анального анастомозов $[4,9]$.

Известные способы профилактики постколэктомического синдрома путем формирования тонкокишечного резервуара в настоящее время применяют исключительно на эта-
\end{abstract}

пе выполнения реконструктивно-восстановительной операции $[3,4,10,11]$. Период между выполнением радикального и реконструктивно-восстановительного этапов операции у таких больных довольно продолжителен, у некоторых из них он составляет от 1 до 3 лет и больше, в связи с трудностями коррекции метаболических нарушений, обусловленных тяжелыми проявлениями постколэктомического синдрома $[8,12]$. Учитывая изложенное, разработка эффективных методов профилактики постколэктомического синдрома на этапе выполнения радикального оперативного вмешательства при двухэтапных операциях представляется чрезвычайно актуальной.

Возникновение синдрома вторичной анальной инконтиненции после формирования илеоанального или резервуарно-анального анастомоза, по данным большинства авторов, обусловлено ендоанальным расположением отрезка тонкой кишки или части тонкокишечного резервуара и, в связи с этим, травмой сфинктерного аппарата прямой кишки $[7,13]$. Поэтому некоторые авторы обоснованно рассматривают конструкцию тонкокишечного резервуара совместно с илеоанальным анастомозом как единую анатомо-функциональную структуру, способную улучшить резервуарную и всасывательную функции тонкой кишки без нарушения функции анального держания [4, 5, 10]. Это обусловливает целесообразность разработки методов, предусматривающих размещение тонкокишечного резервуара на определенном расстоянии от сфинктерного аппарата прямой кишки, а также совершенствование техники формирования илеоанального анастомоза путем ендоанального размещения отрезка тонкой кишки проксимальнее основных порций указанного сфинктерного аппарата прямой кишки $[7,8,13]$.

Значительный риск и сравнительно высокая частота возникновения несостоятельности швов илеоанального, а также резервуарно-анального анастомозов, составляющая 3,4 $18,2 \%$, обусловливают необходимость применения эффективных методов профилактики указанного осложнения [4, 
5, 10], в частности, «отключающей» петлевой илеостомы для противоестественного отведения потока химуса от сформированного тонкокишечного резервуара, илеоанального или резервуарно-анального анастомоза [10, 14 - 16], позволяющей у большинства пациентов предупредить несостоятельность швов, а в случае ее возникновения способствующей уменьшению распространения перитонита и тазового целлюлита $[7,8,13]$. Адекватным хирургическим пособием при возникновении несостоятельности швов илеоанального, резервуарно-анального анастомозов и отсутствии распространенного перитонита является формирование "отключающей" илеостомы, дренирование полости малого таза [3, 4, 11, 14].

Для профилактики некроза тонкокишечного трансплантата чрезвычайно важно сохранить адекватное его кровоснабжение при дислокации в полость малого таза. Для этого необходимо исключить натяжение трансплантата путем мобилизации его брыжейки, компрессию в анальном канале при помощи дивульсии сфинктеров прямой кишки, улучшить микроциркуляцию и перфузию тканей при помощи инфузионной терапии с применением коллоидных трансфузионных сред, низкомолекулярных декстранов, улучшающих реологические свойства крови, а также препаратов, улучшающих процессы микроциркуляции и перфузии тканей [8].

При возникновении некроза низведенного тонкокишечного трансплантата выполняют резекцию некротически измененного участка тонкой кишки, формируют илеостомy $[6,8,17]$.

Для коррекции стриктуры илеоанального анастомоза применяют дозированную его дилатацию, выполняют трансанальное рассечение рубцовых тканей стриктуры с последующей дозированной дилатацией $[8,18]$, реканализацию анастомоза с использованием метода лазерной деструкции рубцовых тканей [8].

Профилактика и лечение осложнений реконструктивновосстановительных операций после колэктомии и предельно низкой передней резекции прямой кишки представляют сложную задачу в связи с высоким риском их возникновения, выраженными метаболическими нарушениями, обусловленными агрессивным течением основного заболевания, обширностью и травматичностью радикального этапа оперативного вмешательства, неблагоприятными условиями заживления илеоанального и резервуарно-анального анастомозов.

Известные и широко используемые в прошлом критерии оценки результатов хирургического лечения, такие как частота возникновения и тяжесть течения ранних и поздних послеоперационных осложнений, рецидивов заболевания, послеоперационная летальность, функциональные результаты, достаточно объективно отражали эффективность проведенного лечения и возможности самореализации оперированного пациента, однако они касались только отдельных аспектов хирургического лечения и не давали целостной оценки его результатов.

Исследование качества жизни оперированных больных, по мнению большинства авторов, представляет новый уровень изучения и оценки результатов проведенного лечения, поскольку учитывает и обобщает все основные функции жизнедеятельности человека, а именно, физическую, психическую, социальную и духовную [7, 19 - 22], то есть позволяет не только дать объективную оценку адаптации пациента к новым анатомическим условиям, возникшим после выполненного сложного оперативного вмешательства, но и определить степень его реинтеграции в общество [7, 21 - 23]. Кроме этого, при помощи критериев качества жизни проводят подбор индивидуальных схем лечения [19, 24], профилактики заболеваний [3], оценивают их эффективность [19, 25, 26]. Технологии определения качества жизни достаточно объективны в связи с непосредственным участием пациента в оценке результатов проведенного лечения [19, 26, 27].
Самым распространенным и наиболее эффективным методом оценки качества жизни в настоящее время является опрос респондентов по стандартизованным схемам с последующей балльной оценкой полученных ответов и подсчетом количества баллов. Выделяют два основных типа опросников, используемых для определения качества жизни: специальные и общие $[19,20,22]$. Среди специальных опросников для оценки качества жизни оперированных больных на органах пищеварения используют гастроинтестинальный индекс качества жизни (Gastroitestinal Quality of Life Index - GIQLI), а также индекс благополучия хирургического больного (Well-Beind-Index for Surgical Patients - WISP) [19, 26]. Однако наибольшее распространение в практике медицинских исследований качества жизни получили общие опросники, в частности опросник Medical Outcomes Study Shoet Form 36 (SF-36) [23, 25], разработанный Бостонским институтом здоровья в рамках международного проекта изучения качества жизни [22]. Он официально зарегистрирован и рекомендован к применению в большинстве стран мира в связи с тем, что охватывает все компоненты качества жизни и наиболее соответствует стандартам, используемым для проведения групповых исследований [22].

Качество жизни пациента, оперированного по поводу тяжелых неопухолевых заболеваний толстой кишки, в значительной степени зависит от уровня послеоперационной реабилитации, которая заключается в физической и психоэмоциональной адаптации к последствиям обширных радикальных операций и особенно к созданным новым тонкокишечным тазовым резервуарным конструкциям [12]. Для этого необходимо понимание пациентом сущности и особенностей клинического течения заболевания, осознание изменений анатомии пищеварительного канала, возникших вследствие выполненной колэктомии, предельно низкой передней резекции прямой кишки, мукозэктомии хирургического анального канала, формирования сложной тонкокишечной тазовой резервуарной конструкции, функциональных ее особенностей $[8,12]$. Уровень реабилитации может быть достаточно высоким, позволяющим пациенту вернуться к полноценной жизни, которой он жил до возникновения заболевания. Для этого необходим этапный мониторинг состояния оперированного больного с целью своевременной диагностики и лечения поздних послеоперационных осложнений: кишечных свищей, стриктуры анастомоза, резервуарита, мегарезервуара, вторичной анальной инконтиненции [8, 12]; соблюдение рекомендаций в отношении образа жизни, диеты, режима питания до завершения периода адаптации, достижения и стабилизации ожидаемых функциональных результатов [12].

Социально-трудовая реабилитация также является чрезвычайно важным критерием качества жизни оперированных больных по поводу неопухолевых заболеваний толстой кишки в связи с тем, что она свидетельствует о степени реализации пациентами индивидуальных социальных программ: приобретения специальности, создания семьи, рождения и воспитания детей, реализации собственного творческого потенциала или остаточной трудоспособности.

К числу основных факторов, которые оказывают отрицательное влияние на качество жизни и реабилитацию пациентов, оперированных по поводу неопухолевых заболеваний толстой кишки, следует отнести наличие илеостомы, рецидива язвенного колита или болезни Крона, семейного аденоматозного полипоза, многоэтапные операции, возникновение поздних послеоперационных осложнений, патологических синдромов, в частности диарейного, постколэктомического, вторичной анальной инконтиненции, для профилактики и лечения которых необходимо применение целенаправленных организационных, тактических мероприятий, совершенствование технических аспектов выполнения реконструктивно-восстановительных операций. 
Таким образом, данные, изложенные в литературе, позволяют сделать вывод о том, что основными недостатками радикального хирургического лечения больных с неопухолевыми заболеваниями толстой кишки являются тяжелые патологические состояния, в частности, рецидив заболевания, постколэктомический, диарейный синдромы, синдром вторичной анальной инконтиненции вследствие значительного уменьшения резервуарности кишечника, нарушения процессов кишечного пищеварения, всасывания, недостаточной компенсации нарушения указанных функций путем формирования классических тонкокишечных тазовых резервуарных конструкций. Поэтому для совершенствования реконструктивно-восстановительных операций необходима разработка более эффективных способов дислокации тонкокишечного трансплантата в полость малого таза без натяжения его брыжейки, тонкокишечных тазовых резервуарных конструкций, способов формирования илеоанального анастомоза для улучшения процессов сращения и функции анального держания

\section{References}

1. Zherlov GK, Bashirov SR. Rezervuarnyy ileorektal'nyy anastomoz. Khirurgiya. 2001;(9):35-40. [In Russian].

2. Khalif IL. Lechebnaya taktika pri yazvennom kolite. Ross med zhurn. 2006;(3):58-61. [In Russian].

3. Rintala RJ, Lindahl HG. Proctocolectomy and J-pouch ileo-anal anastomosis in children. J pediatr Surg. 2002 Jan;37(1):66-70. PMID: 11781989.

4. Rossi HL, Brand MI, Saclarides TJ. Anal complications after restorative proctocolectomy (J-pouch). Am Surg. 2002 Jul; 68(7): 628 - 630. PMID: 12132747.

5. Rotholtz NA, Picarsky AJ, Wexner SD. Adenocarcinoma ariaing from along the rectal stump after double-stapled ileorectal J-pouch in a patient with ulcerative colitis: the rieed to perform a distal anastomosis. Report of a case. Dis. Colon Rectum. 2001 Aug;44(7):1214 - 1217. PMID: 11535865.

6. Baltaytis YuV. Obshirnye rezektsii tolstoy kishki. Kyiv: Zdorov'ia; 1990. 175 s. [In Russian]

7. Melnyk VM. Rekonstruktyvno-vidnovni operatsii pry vydalenni funktsionalno-aktyvnykh viddiliv tovstoi kyshky. DSci [thesis]. Kyiv; 2008. $41 \mathrm{~s}$ [In Ukrainian].

8. Poyda AI, Mel'nik VM. Vosstanovitel'nye i rekonstruktivno-vosstanovitel'nye operatsii na tolstoy kishke. Kyiv: Izdatel'skiy tsentr «Imidzh Ukrainy»; 2014. 368 s. [In Russian].

9. Rink AD, Vestweber KH. Vulnerability of ileocoecal interposition pouch and colonic J-pouch to postoperative adjuvant radiotherapy. Zentralbl Chir. 2002 sep 1;127(9):769-74. doi: 10.1055/s-2002-33956.

10. Selvaggi F, Giuliani A, Gallo C, Signoriello G, Riegler G, Canonico S. Randomiced, controlled trial to compare the J-pouch and $\mathrm{W}$-pouch configurations for ulcerative colitis in the maturation period. Dis. Colon Rectum. 2000 May; 43(5): 615-20. doi: https://doi.org/10.1007/BF02235573.

11. Shen B, Bennett AE, Navaneethan U, Lian L, Shao Z, Kiran RP, et al. Primary sclerosing cholangitis is associated with endoscopic and histologic inflammation of the distal afferent limb in patients with ileal pouchanal anastomosis. Inflamm Bowel Dis. 2011 Sep; 17(9): 1890-900. doi: $10.1002 / \mathrm{ibd} .21594$
12. Poyda AI, Mehlnik VM. Substantiation of formation and results of application of a new type postcolectomy pelvic small intestinal anatomo functional reservoire construction after extremely low anterior rectal resection and mucosectomy. Part 2. Klin khir. 2016;(5):5-9. [In Russian].

13. Melnik VM. Vybor sposoba formirovaniya ileoanal'nogo anastomoza. Kharkivska khirurhichna shkola. 2008;(4):11-5. [In Russian].

14. Chang S, Shen B, Remzi F. When not to pouch: important considerations for patient selection for ileal pouch-anal anastomosis. Gastroenterol Hepatol (N Y). 2017 Aug;13(8):466-475. PMCID: PMC5572960.

15. Shamberger RC, Hergrueter CA, Lillehei CW. Use of a gracilis muscle flap to facilitate delayed ileal pouch-anal anastomosis. dis. colon rectum. 2000 Nov;43(11):1628-31. doi: 10.1007/BF02236752.

16. Schmidt C, Häuser W, Giese T, Stallmach A. Irritable pouch syndrome is associated with depressiveness and can be differentiated from pouchitis by quantification of mucosal levels of proinflammatory gene transcripts. Inflamm Bowel Dis. 2007 Dec;13(12):1502-8. doi: 10.1002/ibd.20241.

17. Fursevich AM. Anatomicheskoe obosnovanie formirovaniya tazovogo tonkokishechnogo rezervuara posle proktokolektomii. Zdravookhranenie. 2000;(6):13-5. [In Russian].

18. Zakharash MP, Poyda OI, Mel'nik VM, Kucher ND, Mal'tsev VN. Khirurgicheskaya taktika pri vosstanovitel'nykh operatsiyakh na tolstoy kishke. Khirurgiya. 2006;(7):51-5. [In Russian].

19. Kanshin NN. Total'naya kolektomiya s odnomomentnoy ileorektostomiey pri tyazhelom nespetsificheskom yazvennom kolite, oslozhnennom suzheniem pryamoy kishki. Vestn. khirurgii im. I. I. Grekova. 2004;163(2):946. [In Russian].

20. Karpukhin OYu, Mozhanov EV, Kolomenskiy SN, Cherenkov EV, Gil'mutdinov AA, Slivochkina NS, Shakurov AF. K voprosu o lechenii bolezni Krona u vzroslykh. Koloproktologiya (prilozhenie). 2011;3(37):130. [In Russian].

21. Karyakina LI. Znachenie optimizatsii metodov diagnostiki i lecheniya VZTK (NYaK i BK) v Vostochno-Kazakhstanskoy oblasti. In: Aktualnie voproci koloproktologii. Materialy II s"ezda koloproktologov. Ufa; 2007. 442-3 s. [In Russian].

22. Navruzov SN, Yusupbekov AA, Khudayarov AE, et al. Khirurgicheskaya taktika pri nespetsificheskom yazvennom kolite. Khirurgiya. 2003;(4):1924. [In Russian].

23. Aghdaei HA, Ghasemi F, Nooraliee M, Fazeli MS, Anaraki F, Sorrentino D, et al. Detailed analysis of total colectomy on health-related quality of life in adult patients with ulcerative colitis. Gastroenterol Hepatol Bed Bench, 2017 Jan; 10 (Suppl. 1):27-32. PMID: 29511468.

24. Yan-Cheng Dai, Lie Zheng, Ya-Li Zhang, Xuan Chen, De-Liang Chen, Zhi-Peng Tang. Effects of Jianpi Qingchang decoction on the quality of life of patients with ulcerative colitis. A randomized controlled trial. Medicine, 2017 Apr; 96(16):e6651. doi: 10.1097/MD.0000000000006651.

25. Paweł Kawalec, Ewa Stawowczyk, Małgorzata Mossakowska, Andrzej Pilc. Disease activity, quality of life, and indirect costs of ulcerative colitis in Poland. Gastroenterology Rev 2017 Mar 7;12(1):60-5. doi: 10.5114 pg.2017.66324.

26. Tajti János Jr, Látos Melinda, Farkas Klaudia, Ábrahám Szabolcs, Simonka Zsolt, Paszt Attila, et al. Effect of Laparoscopic Surgery on Quality of Life in Ulcerative Colitis. J Laparoendosc Adv Surg Tech A. 2018 Jan 25. doi: 10.1089/lap.2017.0698.

27. Kravchenko TG. Kachestvo zhizni bol'nykh, operirovannykh po povodu yazvennogo kolita. Liky Ukrainy. 2015;3(24):39-43. [In Russian]. 